\title{
An Emerging Treatment Alternative for Anemia in Chronic Kidney Disease Patients: A Review of Daprodustat
}

\author{
Kimberly A. Becker · John J. Jones
}

Received: October 31, 2017 / Published online: December 28, 2017

(C) The Author(s) 2017. This article is an open access publication

\begin{abstract}
This article reviews an emerging therapeutic agent, which is currently in phase III development for the treatment of anemia secondary to chronic kidney disease, covering promising phase II results, drug characteristics, and the current phase III trials, which, if approved, may significantly impact the management of anemia in this patient population.
\end{abstract}

Keywords: Anemia; Chronic kidney disease; Daprodustat; Dialysis; Erythropoietinstimulating agent; HIF-inhibitor; Hematology; Hemoglobin; Nephrology; Non-dialysis

\section{INTRODUCTION}

More than one in seven Americans are estimated to be living with chronic kidney disease (CKD) [1]. Patients with CKD are prone to developing multiple comorbidities, including

Enhanced content To view enhanced content for this article go to http://www.medengine.com/Redeem/ 6DFCF060605858A5.

\section{K. A. Becker $(\bowtie)$}

Lerin Drug Co. Inc., New York, NY, USA

e-mail: kimberly.anne.becker@gmail.com

J. J. Jones

St. John's University, Jamaica, NY, USA anemia which can become more severe as the disease progresses [2]. Anemia in CKD patients may be caused by iron deficiency, inflammation, or a disruption in the production of erythropoietin (EPO) $[2,3]$. EPO is a hormone that stimulates the production of red blood cells [4]. Deficiencies of this hormone are currently managed with EPO-stimulating agents (ESAs), usually in combination with iron supplementation. However, the FDA has issued warnings on the use of EPO analogs due to risk of death and serious cardiovascular events [5-7]. The cardiovascular safety findings of EPO analogs were based on a number of controlled trials which targeted hemoglobin $(\mathrm{Hb})$ values greater than $11 \mathrm{~g} / \mathrm{dL}[8,9]$. The warnings stated that no trial has identified a $\mathrm{Hb}$ target level, ESA dose, or dosing strategy that does not increase these risks. The FDA labeling recommends using the lowest EPO analog dose sufficient to reduce the need for red blood cell (RBC) transfusions [5-7].

Daprodustat is an orally administered, smallmolecule hypoxia-inducible factor prolyl hydroxylase (HIF-PH) inhibitor currently in phase III development [10]. The expected completion dates of the phase III trials are 2018 and 2019 for US approval. This agent offers a novel approach to managing anemia in CKD patients that could affect clinical practice recommendations and guidelines. This article is based on previously conducted studies and does not involve any new studies of human or animal subjects performed by the authors. 


\section{SCIENTIFIC SUMMARY}

\section{Mechanism of Action}

When the body is exposed to low oxygen levels, such as at increased altitude, the body stimulates the production of EPO to increase RBCs and restore oxygen balance $[3,11]$. This mechanism of compensation is initiated through the transcription factor, HIF- $\alpha$, more specifically the isoform, HIF- $1 \alpha$, which is commonly referred to as HIF-1. When HIF- $\alpha$ accumulates, it forms a functional dimer with HIF- $\beta$, which subsequently translocates to the nucleus where it binds to hypoxia response elements (HREs) on DNA. This results in the induction of gene expression of EPO, which in turn promotes an erythropoietic response and upregulation of iron transport $[3,12]$. Iron is transferred from iron stores in the liver and from intestinal mucosal cells in response to a decrease in hepcidin, a hormone that indirectly regulates iron. Iron is carried by a protein called transferrin so it can be mobilized to the bone marrow.

When the body has adequate oxygen levels, HIF- $\alpha$ is degraded by a regulatory enzyme, HIFPH [3, 12]. Daprodustat stabilizes HIF- $1 \alpha$ by inhibiting HIF-PH, therefore increasing levels of HIF- $\alpha$, leading to increases in EPO and decreases in hepcidin $[3,11,12]$.

\section{Pharmacokinetics (PK)}

\section{Metabolism}

Daprodustat is a highly protein-bound molecule and undergoes oxidative metabolism primarily in the liver by the CYP2C8 enzyme into 6 metabolites with an approximate half-life of up to $4 \mathrm{~h}$ (100-mg dose) [12].

\section{Absorption \\ Results from a study designed to characterize differences in PK/PD parameters between Japa- nese and Caucasian participants revealed varia- tions between groups. Absorption of daprodustat with a median concentration peak time $\left(t_{\max }\right)$ was $1.3-2.5 \mathrm{~h}$ in the Japanese group and $1.0-2.0 \mathrm{~h}$ in the Caucasian group [13]. Across doses of $10,25,50$, and $100 \mathrm{mg}$, the}

mean terminal half-life $\left(t_{1 / 2}\right)$ ranged from 1.1 to $2.3 \mathrm{~h}$ in the Japanese group and from 0.9 to $1.9 \mathrm{~h}$ in the Caucasian group [13]. Higher exposure of the drug determined by $C_{\max }$ and AUC $_{\text {inf }}$ was observed in the Japanese group relative to the Caucasian group [13]. The clinical significance of these PK differences have been studied in a phase II trial and are being further evaluated in an ongoing phase III trial.

\section{Potential Interactions}

Co-administration of a single dose of daprodustat with a high-fat/high-calorie meal did not significantly impact plasma AUC; however, a $29 \%$ mean reduction of $C_{\max }$ was observed. [12]. It is unknown whether this will translate into clinical relevance; thus, in order to minimize drug exposure variability within patients, investigators recommended consistent administration of daprodustat either with food or without food. Concomitant administration with steady-state gemfibrozil, a strong CYP2C8 inhibitor, resulted in significantly increased $\mathrm{AUC}_{\mathrm{inf}}, C_{\max }$, and $t_{1 / 2}$ of daprodustat, which suggests that significant drug-drug interactions will occur with CYP2C8 inhibitors [12].

\section{Pharmacodynamics (PD)}

Daprodustat demonstrated a dose-dependent increase in a variety of pharmacodynamic markers, including EPO concentration, absolute reticulocyte count, $\mathrm{Hb}$ level, and total ironbinding capacity (TIBC), an indirect measurement of the iron-binding capacity of transferrin in the blood $[14,15]$. Conversely, daprodustat demonstrated a dose-dependent decrease in hepcidin and ferritin (storage iron), which signifies utilization of iron stores $[14,15]$. Surrogate markers of pharmacodynamic effects, including EPO, VEGF, and absolute reticulocyte counts, have also exhibited dose-ordered increases over a range of 10-100 mg [13]. VEGF, which is directly regulated by HIF1 and is implicated in angiogenic activity, did increase post-dose; however, there have been inconsistent variabilities between placebo and the study drug to conclude whether these increases will carry clinical significance $[13,14,16]$. 


\section{Phase I Trials}

The safety and tolerability of repeat doses of daprodustat in 151 adult patients have been evaluated in several phase I studies [10]. Doses from 2 to $450 \mathrm{mg}$ have been assessed in healthy volunteers, and $5 \mathrm{mg}$ was studied in a mixed population of healthy and renally impaired participants as well as in patients undergoing peritoneal dialysis (PD). Cardiac repolarization has been studied with a dose of $500 \mathrm{mg}$.

\section{PHASE II THERAPEUTIC TRIALS}

\section{Management of Anemia in Non-Dialysis- Dependent (NDD) and HD CKD Patients}

\section{Studies 1 and 2: 0.5, 2, and $5 \mathrm{mg}$}

Two parallel phase IIA randomized, blinded, controlled studies were conducted with daprodustat over a 4-week time period in separate populations with CKD to evaluate the safety and efficacy of daprodustat [14]. The first study, referred to as the 'Nondialysis Study', was conducted in patients with CKD stages 3-5 not undergoing dialysis who had not used recombinant human erythropoietin (rhEPO) within the past 7 weeks. The second study, referred to as the 'Hemodialysis Study', was conducted in patients receiving hemodialysis three times weekly and adequately responded to rhEPO treatment. Per the protocol, patients would be withdrawn from the studies if their hemoglobin was $<8.0,>13.0 \mathrm{~g} / \mathrm{dL}$, or they experienced a change of $2.0 \mathrm{~g} / \mathrm{dL}$ in a 1 -week time period. Patients were randomized 1:1:1:1 in each study with the active treatment being one of three doses of daprodustat, $0.5,2$, or $5 \mathrm{mg}$. Placebo was used in the nondialysis study while maintenance of current rhEPO therapy was used as the control arm in the HDD study.

Seventy-three patients were randomized in the NDD study, of which 59 (81\%) had completed the study through follow-up at weeks 6 and 54 [14]. One-hundred percent of the NDD patients had cardiovascular risk factors at baseline, including hypertension (97\%), hyperlipidemia (76\%), and diabetes (69\%). In the HDD study, 83 patients were randomized, of which
$70(84 \%)$ patients completed the study through follow-up. Similarly, 95\% of the dialysis participants had cardiovascular risk factors including hypertension (95\%), hyperlipidemia (57\%), and diabetes (45\%). Over the 4-week treatment period in the NDD study, the mean observed change from baseline (CFB) in hemoglobin for the $0.5,2$, and $5 \mathrm{mg}$ doses of daprodustat were $-0.121,0.121$, and $0.951 \mathrm{~g} / \mathrm{dL}$ respectively, compared to $-0.15 \mathrm{~g} / \mathrm{dL}$ in the placebo arm. In the HDD study, CFB in Hb for doses of $0.5,2$, and $5 \mathrm{mg}$ of daprodustat were $-1.06,-0.93$, and $-0.08 \mathrm{~g} / \mathrm{dL}$ respectively compared to $-0.25 \mathrm{~g} / \mathrm{dL}$ in the active control arm. EPO concentrations did not accumulate over the treatment period with daprodustat. Comparing the median peak EPO concentrations across the groups in the HDD study, the results were $424.9,13.9,12.7$, and $24.7 \mathrm{U} / \mathrm{L}$ for the rhEPO $0.5,2$, and $5 \mathrm{mg}$ of daprodustat groups, respectively. There were no clinically relevant changes in plasma VEGF in either daprodustat studies. The pharmacodynamic assessments were limited to $11 \mathrm{~h}$ post-dose, and thus the true peak plasma concentrations of EPO and VEGF may not have been captured. Five AEs were reported for one or more participants receiving daprodustat: two experienced nausea in the nondialysis study and three experienced anemia in the HDD study. However, drug-related adverse events were not clearly distinguished in these studies. While no deaths occurred in either study, two serious adverse events (SAEs) were reported post-therapy in the HDD study for patients receiving $0.5 \mathrm{mg}$ of daprodustat, which included an abnormal liver function test and acute respiratory failure.

\section{Study 3: 10, 25, 50, and $100 \mathrm{mg}$}

A phase IIA, randomized, placebo-controlled, dose ranging study of daprodustat was conducted in anemic patients $(\mathrm{Hb} \leq 11.0 \mathrm{~g} / \mathrm{dL})$ with CKD [15]. Participants were grouped separately according to their degree of renal impairment; that is, either NDD-CKD stages 3-5 or hemodialysis-dependent CKD stage 5 (corresponding eGFR: $15-59 \mathrm{~mL} / \mathrm{min} / 1.73 \mathrm{~m}^{2}$ for stages $3-4 ; 10$ to $<15 \mathrm{~mL} / \mathrm{min} / 1.73 \mathrm{~m}^{2}$ for stage $5)$. The study evaluated safety and tolerability end points as well as collected PK/PD data for 
once-daily administration of daprodustat over a period of 28 days with post-treatment follow-up to day 57. Participants were enrolled to receive doses of $10,25,50$, and $100 \mathrm{mg}$ in the NDD group and doses of 10 and $25 \mathrm{mg}$ in the HD group. A total of 107 participants, predominantly white females and patients of Asian descent, were enrolled in this study. Seventy patients were randomized to the NDD group and 37 were randomized to the HD group.

A priori response rates were defined as a $\mathrm{Hb}$ level increase of 1.0 and $0.5 \mathrm{~g} / \mathrm{dL}$ [15]. The percentage of patients achieving these response were 63 and 91\% for the NDD group and 71 and $89 \%$ for the HD group. Per the protocol-defined safety criteria, dosing in any treatment arm would be stopped if the $\mathrm{Hb}$ level was $>13.5 \mathrm{~g} /$ $\mathrm{dL}$ or the rate of $\mathrm{Hb}$ level increase was $>1 \mathrm{~g} / \mathrm{dL}$ over a 2-week period. Combined, the number of patients that experienced a rapid increase in $\mathrm{Hb}$ level and subsequent study withdrawal was 21 of $70(30 \%)$ and 8 of $37(22 \%)$ for the NDD and HD groups, respectively. Accounting for all reasons for withdrawal, only 30 participants in the NDD group and 22 participants in the HD group receiving daprodustat completed the study. With respect to the safety of daprodustat, 35 of 61 patients with NDD-CKD and 15 of 31 patients with HD-CKD experienced AEs, of which 16 were considered by the investigator to be drug-related. Nausea was the most common drug-related AE in the NDD group, with 5 of the 6 events occurring with those receiving the $100 \mathrm{mg}$ dose. While no deaths were reported in the study, 2 of 7 participants reported SAEs in the NDD group occurred in patients receiving 100-mg doses and were considered to be related to the study drug. None of the reported SAEs for the 3 participants in the HD group were considered to be related to daprodustat.

\section{Management of Anemia in Hemodialysis (HD) Patients: 4, 6, 8, and $10 \mathrm{mg}$}

A phase II, randomized, placebo-controlled, dose ranging study of daprodustat was conducted in Japanese patients on HD in response to variabilities in a PK analysis in a previous phase I trial [16]. This study sought to evaluate the $\mathrm{Hb}$ dose response and the safety of daprodustat in Japanese patients $[13,16]$. The study randomized 97
Japanese patients who had been on HD for $\geq 8$ weeks and who were effectively managed with an ESA resulting in stable Hb levels. Patients discontinued their ESA for 2-8 weeks prior to enrollment and were eligible for inclusion if they had a decrease in $\mathrm{Hb}$ of $\geq 0.5 \mathrm{~g} / \mathrm{dL}$ with a resulting baseline of 8.5-10 g/dL. Patients were randomized $1: 1: 1: 1: 1$ to receive $4,6,8,10 \mathrm{mg}$ of daprodustat or placebo daily. Eighty-nine percent of patients completed the trial with the mean baseline $\mathrm{Hb}$ ranging from 9.68 to $9.92 \mathrm{~g} / \mathrm{dL}$. The primary endpoint of change in Hb levels at week 4 was $-0.28,-0.01,0.54$, and 0.97 in the $4,6,8$, and $10 \mathrm{mg}$ groups, respectively, compared to a decrease of $-1.41 \mathrm{~g} / \mathrm{dL}$ in the placebo arm. Changes in other blood parameters such as RBC and reticulocytes were consistent with the changes in $\mathrm{Hb}$. Plasma EPO concentrations increased in a dose-related manner up to $8 \mathrm{mg}$, with no additional increases with $10 \mathrm{mg}$. HIF-1 upregulates VEGF, which is implicated in angiogenic activity, tumor growth, and macular edema. VEGF levels were also measured and there were no significant differences observed between any of the groups.

Other secondary endpoints included iron parameters [16]. Totals of 5 and $20 \%$ of participants received stable oral or IV iron supplementation, respectively, throughout the study. There was a dose-dependent decrease in hepcidin and ferritin coupled with increases in transferrin and TIBC, indicating a dose-response relationship with increased iron utilization. Modest decreases in lipid levels were seen at week 4 in the daprodustat arm, and there was a higher incidence of nasopharyngitis in participants who received daprodustat compared to placebo.

\section{EXPLORATORY THERAPEUTIC INDICATIONS INVESTIGATED}

\section{Management of Claudication-Limited Peripheral Artery Disease}

It has been hypothesized that modulating oxygen delivery in patients with peripheral artery disease (PAD) could play a critical role in the treatment of ischemic diseases [17]. Preclinical 
data suggest that patients with ischemic diseases have reduced HIF activity. This multicentered, randomized, placebo-controlled study tested whether administration of daprodustat as a 300-mg single-dose followed by a 15-mg daily dose over the course of 2 weeks would affect performance measures in these patients. PK/PD studies were evaluated pre- and post-dosing, and a muscle biopsy was preformed to assess changes in concentration of HIF gene expression in the muscle. Participants over 40 years old with PAD, defined as having an ankle-brachial index $(\mathrm{ABI}) \leq 0.90 \mathrm{in}$ at least $1 \mathrm{leg}$ at rest for at least 3 months, were eligible for the study. Forty-six patients were recruited with no differences in baseline characteristics between active and controlled groups. The most common adverse events seen in the daprodustat group were pain in the extremity, diarrhea, dyspepsia, upper respiratory infections, and fatigue. Eight percent of patients in the daprodustat experienced SAEs of arrhythmias, congestive heart failure, chronic obstructive pulmonary disease, or hyponatremia. There were no differences in improvement in the 6-min walk test or the bilateral heel rise test. Per PK analysis, there was an increase in EPO after the first dose, but there were no differences in EPO levels on subsequent doses compared to placebo. There were increases in $\mathrm{Hb}$ after the subsequent doses of daprodustat were administered. Compared to the placebo group, participants in the daprodustat group exhibited decreases in total cholesterol. For the participants who underwent muscle biopsy, there were no increases in mRNA or proteins of HIF target genes.

The HIF response mechanism was effectively targeted in this patient population, but it did not induce the anticipated clinical response [17].

\section{SAFETY}

Daprodustat appears to be well tolerated as evidenced by the most commonly reported side effect being nausea $[14,15]$. However, due to protocol-defined safety criteria, a considerable number of patients were withdrawn from these studies because of a robust pharmacodynamic $\mathrm{Hb}$ response, indicating a potential for long- term cardiovascular and/or malignant events that have yet to be uncovered given the short time frame for these trials $[14,15]$.

\section{ONGOING CLINICAL TRIALS}

Currently, there are four phase III clinical trials evaluating the efficacy and safety of daprodustat in a total of 8,070 patients with varying degrees of CKD, including dialysis dependency and non-dialysis dependency (Table 1) [10]. The trials have parallel assignment study designs against an active comparator, namely, darbepoetin alfa, which is an ESA as well as a rhEPO. The primary efficacy endpoints for these trials include measuring mean $\mathrm{Hb}$ between baseline and the efficacy period, varying from weeks 40-52 and weeks 28-52, and an event-driven time to first occurrence of adjudicated major adverse cardiovascular event [composite of allcause mortality, non-fatal myocardial infarction (MI) and non-fatal stroke]. Results from the composite endpoints in the ASCEND trials will provide clinically meaningful comparative safety data for daprodustat.

\section{CONCLUSIONS}

According to the existing body of evidence from the published literature, daprodustat has thus far demonstrated the potential to be an effective alternative for the treatment of anemia in patients with CKD [14-16]. Daprodustat has exhibited dose-dependent EPO and non-EPO response mechanisms for effectively increasing and maintaining $\mathrm{Hb}$ levels. There were no clinically relevant increases in VEGF compared to placebo groups, which signifies that the regulation of VEGF by EPO may not be influenced by the inhibition of HIF-PH. A significant percentage of patients were withdrawn due to increases in $\mathrm{Hb} \geq 1 \mathrm{~g} / \mathrm{dL}$ or a $\mathrm{Hb}$ level $>13.5 \mathrm{~g} /$ $\mathrm{dL}$ over a 2-week period, as specified by the protocol [14]. These robust pharmacodynamic responses over such short time periods may indicate a potential for cardiovascular or carcinogenic side effects with daprodustat when examined over a longer time period. Common 
Table 1 Ongoing phase III daprodustat trials

\begin{tabular}{|c|c|c|c|}
\hline Trial name & Comparator(s) & $\begin{array}{l}\text { ClinicalTrials.gov } \\
\text { identifier }\end{array}$ & $\begin{array}{l}\text { Estimated primary } \\
\text { completion date; } \\
\text { estimated enrollment }\end{array}$ \\
\hline $\begin{array}{l}\text { A Study to Evaluate Efficacy and Safety of } \\
\text { Daprodustat Compared to Darbepoetin Alfa in } \\
\text { Japanese Hemodialysis (HD)-Dependent Subjects } \\
\text { With Anemia Associated With Chronic Kidney } \\
\text { Disease (CKD) }\end{array}$ & $\begin{array}{l}\text { Darbepoeitin } \\
\text { alfa }\end{array}$ & NCT02969655 & July 2018; 270 patients \\
\hline $\begin{array}{l}\text { A Study to Evaluate the Efficacy and Safety of } \\
\text { Daprodustat Compared to Recombinant Human } \\
\text { Erythropoietin (rhEPO) in Subjects With Anemia } \\
\text { Associated With Chronic Kidney Disease (CKD) } \\
\text { Who Are Initiating Dialysis }\end{array}$ & $\begin{array}{l}\text { Darbepoeitin } \\
\text { alfa }\end{array}$ & NCT03029208 & $\begin{array}{l}\text { November 2019; } 300 \\
\text { patients }\end{array}$ \\
\hline $\begin{array}{l}\text { Anemia Studies in Chronic Kidney Disease: } \\
\text { Erythropoiesis Via a Novel Prolyl Hydroxylase } \\
\text { Inhibitor Daprodustat-Dialysis (ASCEND-D) }\end{array}$ & rhEPO & NCT02879305 & April 2020; 3000 patients \\
\hline $\begin{array}{l}\text { Anemia Studies in Chronic Kidney Disease: } \\
\text { Erythropoiesis Via a Novel Prolyl Hydroxylase } \\
\text { Inhibitor Daprodustat-Non Dialysis (ASCEND- } \\
\text { ND) }\end{array}$ & $\begin{array}{l}\text { Darbepoetin } \\
\text { alfa }\end{array}$ & NCT02876835 & $\begin{array}{l}\text { January } 2021 ; 4500 \\
\text { patients }\end{array}$ \\
\hline
\end{tabular}

side effects such as nausea and dyspepsia were well tolerated and were mostly benign $[14,15]$. Based on the exploratory clinical trial, daprodustat is ineffective for the treatment of PAD [17]. These data are primarily limited based on the length of the studies and small population sizes. Lastly, there are other HIF-PH inhibitors currently in phase III of development. While these agents share the same mechanism of action, they vary with respect to dosing and administration [10]. If the phase III trials for daprodustat reveal positive results, it will be one of four HIF-PH inhibitors seeking FDA approval for the treatment of anemia in patients with $\mathrm{CKD}$ in the upcoming years.

\section{ACKNOWLEDGEMENTS}

Funding. No funding or sponsorship was received for this study or publication of this article.
Authorship. All named authors meet the International Committee of Medical Journal Editors (ICMJE) criteria for authorship for the manuscript, take responsibility for the integrity of the work as a whole, and have given final approval for the version to be published.

Disclosures. Kimberly Becker and John Jones have nothing to disclose.

Compliance with Ethics Guidelines. This article is based on previously conducted studies and does not involve any new studies of human or animal subjects performed by the authors.

Data Availability. Data sharing is not applicable to this article as no datasets were generated or analyzed during the current study.

Open Access. This article is distributed under the terms of the Creative Commons Attribution-NonCommercial 4.0 International License (http://creativecommons.org/licenses/ by-nc/4.0/), which permits any non- 
commercial use, distribution, and reproduction in any medium, provided you give appropriate credit to the original author(s) and the source, provide a link to the Creative Commons license, and indicate if changes were made.

\section{REFERENCES}

1. Centers for Disease Control and Prevention. Chronic Kidney Disease. CDC.gov. https://nccd. cdc.gov/ckd/. Accessed Sep 18, 2017.

2. Stauffer ME, Fan T. Prevalence of anemia in chronic kidney disease in the United States. PLoS ONE. 2014;9(1):e84943.

3. Gupta N, Wish JB. Hypoxia-inducible factor prolyl hydroxylase inhibitors: a potential new treatment for anemia in patients with CKD. Am J Kidney Dis. 2017;69(8):815-26.

4. Onken J, Bregman B, Harrington RA, et al. Ferric carboxymaltose in patients with iron deficiency anemia and impaired renal function: the REPAIRIDA trial. Nephrol Dial Transplant. 2013;29:833-42.

5. Epogen [package insert]. Amgen Inc., Thousand Oaks, CA; 1989.

6. Procrit [package insert]. Amgen Inc., Thousand Oaks, CA: 1989.

7. Aranesp [package insert]. Amgen Inc., Thousand Oaks, CA; 2001.

8. Pfeffer MA, Burdmann EA, Chen CY, et al. A trial of darbepoetin alfa intype 2 diabetes and chronic kidney disease. N Engl J Med. 2009;361(21):2019-32.

9. Drüeke TB, Locatelli F, Clyne N, et al. Normalization of hemoglobin level in patients with chronic kidney disease and anemia. $\mathrm{N}$ Engl J Med. 2006;355(20):2071-84.

10. U.S. National Institutes of Health. HIF inhibitor. ClinicalTrials.gov. http://www.clinicaltrials.gov. Accessed Sep 18, 2017.

11. Becker K, Saad M. A new approach to the management of anemia: a review on roxadustat. Adv Ther. 2017;34:848-53.

12. Johnson BM, Stier BA, Caltabiano S. Effect of food and gemfibrozil on the pharmacokinetics of the novel prolyl hydroxylase inhibitor GSK1278863. Clin Pharmacol Drug Dev. 2014;3(2):109-17.

13. Hara K, Takahashi N, Wakamatsu A, Caltabiano S. Pharmacokinetics, pharmacodynamics and safety of single, oral doses of GSK1278863, a novel HIFprolyl hydroxylase inhibitor, in healthy Japanese and Caucasian subjects. Drug Metab Pharmacokinet. 2015;30:410-8.

14. Holdstock L, Meadowcroft AM, Maier R, et al. Fourweek studies of oral hypoxia-inducible factor-prolyl hydroxylase inhibitor GSK1278863 for treatment of anemia. J Am Soc Nephrol. 2016;27:1234-44.

15. Brigandi R, Johnson B, Oei C, et al. A novel hypoxia-inducible factor-prolyl hydroxylase inhibitor (GSK 1278863) for anemia in CKD: a 28-day, phase $2 \mathrm{~A}$ randomized trial. Am J Kidney Dis. 2016;67(6):861-71.

16. Akiwaza T, Tsubakihara Y, Nangaku M, et al. Effects of daprodustat, a novel hypoxia-inducible factor prolyl hydroxylase inhibitor on anemia management in japanese hemodialysis subjects. Am J Nephrol. 2017;45:127-35.

17. Olson E, Demopoulos L, Haws TF, et al. Short-term treatment with a novel HIF-prolyl hydroxylase inhibitor (GSK1278863) failed to improve measures of performance in subjects with claudication-limited peripheral artery disease. Vasc Med. 2014;19(6):473-82. 